\title{
Automatic IP Generation of FFT/IFFT Processors with Word-Length Optimization for MIMO-OFDM Systems
}

\author{
Pei-Yun Tsai, Chia-Wei Chen, and Meng-Yuan Huang \\ Department of Electrical Engineering, National Central University, Jhongli 32001, Taiwan \\ Correspondence should be addressed to Pei-Yun Tsai, pytsai@ee.ncu.edu.tw
}

Received 26 May 2010; Revised 18 October 2010; Accepted 11 November 2010

Academic Editor: Juan A. López

Copyright (C) 2011 Pei-Yun Tsai et al. This is an open access article distributed under the Creative Commons Attribution License, which permits unrestricted use, distribution, and reproduction in any medium, provided the original work is properly cited.

A systematic approach is presented for automatically generating variable-size FFT/IFFT soft intellectual property (IP) cores for MIMO-OFDM systems. The finite-precision effect in an FFT processor is first analyzed, and then an effective word-length searching algorithm is proposed and incorporated in the proposed IP generator. From the comparison, we show that our analysis of the finite precision effect in FFT is much more accurate than the previous work. With the flexible architecture and the effective word-length searching techniques, we can strike a good balance for the performance and the hardware cost of the generated IP cores. The generated FFT soft IP cores are portable and independent of the silicon technology, which helps to greatly reduce the design time. Experimental results demonstrate that the proposed IP generator indeed provides FFT IPs which meet the requirements and are more suitable in recent MIMO-OFDM communication standards/drafts than some conventional FFT IP generators.

\section{Introduction}

Orthogonal frequency-division multiplexing (OFDM) is one of the most popular modulation schemes in recent wireless communication systems. In OFDM transceivers, discrete Fourier transform (DFT) operation plays an important role to modulate data onto each subcarrier. With the fast Fourier transform (FFT) algorithm, hardware implementation of DFT, which is not only computation intensive but also communication intensive, becomes feasible.

Different OFDM systems use various FFT sizes to accommodate time-selective and/or frequency-selective channel environments. Even in one system, FFT operations of variable sizes are mandatory to offer the scalability for performance considerations. In addition, multiple-input multipleoutput (MIMO) antenna configuration is a widely adopted technique recently, which needs a multichannel FFT/IFFT processor in a transmitter/receiver. An extensive literature exists, which reports the lower-power/small-area/high-speed implementation of the dedicated FFT processors for certain single-input single-output (SISO) wireless communication standards/specifications $[1-5]$ and for multiple-input multiple-output OFDM systems $[6,7]$.
However, it is a time-consuming work if a dedicated FFT processor is redesigned each time for every communication system. In the past, several general-purpose FFT IP core generators have also been developed [8-11] including the state-of-the-art spiral program $[12,13]$. On the other hand, FFT/IFFT core generators specific for OFDM systems can be seen in $[14,15]$. In $[11,12,15]$, the generated hardware employs the radix-2 FFT algorithm and different degrees of parallelism are exploited, either using multiple butterfly stages or multiple butterfly units inside a butterfly stage, to tradeoff throughput requirements and hardware costs. Radix- 2 and radix-4 pipelined multipath delay commutator architectures have been used in $[8,9]$. Higher radix algorithm (radix-2/4/8) was first utilized in [14], which adopts memory-based architecture and pipelined single-path delay feedback architecture. Note that the FFT/IFFT core generator in [15-17] is capable of generating an FFT IP that handles variable-size FFT/IFFT operations and satisfies the signal-toquantization-noise-ratio (SQNR) constraint.

In this paper we propose an IP generator to offer userspecific FFT processors targeting at the requests in recent and emerging MIMO-OFDM communication systems. However, 
different from previous works, we try to analyze the finite precision effect in FFT processors and aim to offer an FFT IP generator that has the capability of automatic wordlength optimization to achieve hardware efficiency. The IP generator can generate the hardware description language of an FFT processor according to the constraints set by users and therefore speed up the process for implementing a new OFDM transceiver. Its features can be summarized as follows.

(i) Parallel processing and multiple channels are taken into consideration, either to increase throughput or to support MIMO configurations.

(ii) The word lengths are optimized, which can be shown to provide more efficient hardware design under the constraint of SQNR values than some conventional works $[15,16]$.

(iii) Insertion of pipeline registers mainly depends on the requirement of operating frequency to ensure the necessity of flip-flop instantiation.

From the experimental results, we can see that these improvements are effective to generate FFT IPs that strike a good balance between complexity and performance.

The rest of the paper is organized as follows. In Section 2, the generic FFT architecture adopted by the proposed FFT IP generator is illustrated. In Section 3, we discuss the finite precision effect in FFT operation. The work flow of the IP generator and the word-length optimization procedure are delineated in Section 4. Experimental results and comparisons are shown in Section 5. Finally, Section 6 gives a brief conclusion.

\section{Architecture of FFT Processors with MIMO Configuration and Parallel Processing}

In Table 1, we have listed some essential parameters in several recent OFDM standards/drafts. Note that in UWB using MB-OFDM modulation scheme, we show its one-channel sampling rate. It is clear that the needed FFT processor must support variable sizes as well as parallel processing for either high throughput or multiple channels. In addition, the FFT sizes mainly range from 64 points to 8192 points, and the operating frequency covers from tens to hundreds of mega Hz. To facilitate automatic generation of the FFT processors fulfilling the above requirements, we resort to exploit the mapping of its recursive nature to the pipelined architecture. However, to accomplish parallel processing with the high-radix algorithm, we proposed to combine two well-known pipelined architectures, namely, the single-path delay feedback (SDF) architecture and the multipath delay commutator (MDC) architecture.

Figure 1 shows our adopted architecture that is able to support the parallelism degree of two or four by utilizing the property of the multipath delay commutator architecture in parallel processing. If the parallelism degree of $p$ is desired, where $p=2$ or 4 , a radix- $p$ MDC stage is first employed. Thereafter, for the $p$ parallel paths, we cascade $p$-channel $N / p$-point FFT processors implemented

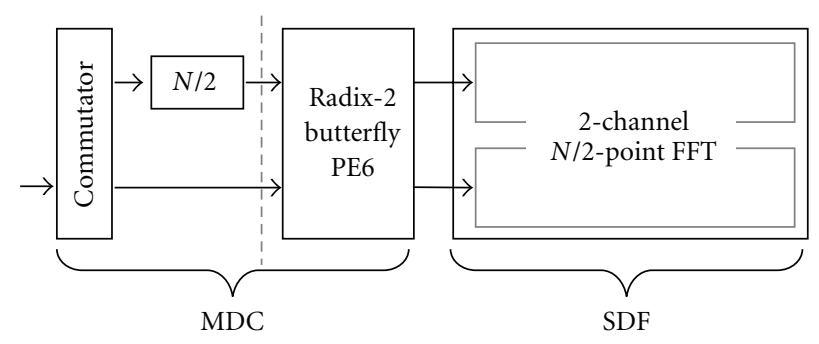

(a)

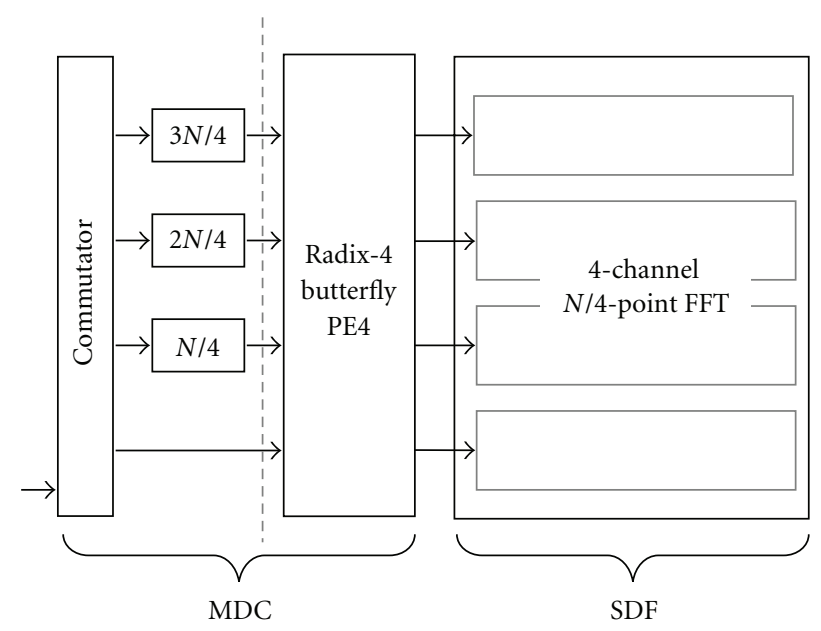

(b)

FIgure 1: (a) Architecture of an FFT processor with parallelism degree of two. (b) Architecture of an FFT processor with parallelism degree of four.

by the radix $-2 / 2^{2} / 2^{3}$ single-path delay feedback architecture. If parallel processing to enhance the throughput is not necessary, the generated FFT processor is reduced to the conventional SDF architecture.

Table 2 compares the hardware complexity of the proposed architecture and several conventional works with parallelism [3, 18-24]. However, those works may be designed for specific applications such as UWB and may have special optimization at certain stages. Here, we simply consider their extensions to an $N$-point FFT processor. Note that hardware complexity and architecture flexibility are essential concerns. In our adopted architecture of parallelism degree of two, one complex multiplier is required in the first radix-2 MDC processing element and $2\left(\log _{8}(N / 2)-1\right)$ complex multipliers are used in the remaining two sets of radix- $2^{3} N / 2$-point SDF architecture. Similarly, if the parallelism degree is four, $3+4\left(\log _{8}(N / 4)-1\right)$ complex multipliers are needed in our architecture instead of $3\left(\log _{4} N-1\right)$ complex multipliers in the conventional radix-4 MDC architecture. Although the higher radix $-2^{4}$ architecture [20, 23] can effectively reduce the number of complex multipliers, the constant multipliers increase. Special scheduling for some specific FFT size can help to decrease the complexity of the constant multipliers [19]. Nevertheless it is not easily provided in an IP generator offering diverse user-specific parameters. Also the folding scheme (SDF-kR) is not appropriate because higher and 
TABLE 1: FFT parameters in several OFDM systems..

\begin{tabular}{lccc}
\hline & FFT size & Sampling rate (MHz) & MIMO channels \\
\hline DVB-T/H & $2048-8192$ & 9 & - \\
$802.11 \mathrm{a}$ & 64 & 20 & - \\
$802.11 \mathrm{n}$ & $64-128$ & 40 & Up to 4 \\
UWB (MB-OFDM) & 128 & 528 & 2 \\
802.16 (OFDM) & 256 & 32.7 & - \\
802.16 (OFDMA) & $128-2048$ & 20 & Up to 4 \\
3GPP-LTE & $128-2048$ & 30.7 & Up to 4 \\
802.20 & $512-2048$ & 20 & Up to 4 \\
\hline
\end{tabular}

TABLE 2: Complexity comparison of several FFT processors with parallelism.

\begin{tabular}{|c|c|c|c|c|c|c|}
\hline Parallelism & Architecture & Complex multipliers & Constant multipliers & Storages & Clock rate & Throughput \\
\hline 2 & Radix-2 MDC & $\log _{2} N-1$ & - & $\frac{3 N}{2}-2$ & $\mathrm{R}$ & $2 \mathrm{R}$ \\
\hline 2 & Radix-2 $2^{2}$ MDF [18] & $\log _{2} N-2$ & - & $N-2$ & $\mathrm{R}$ & $2 \mathrm{R}$ \\
\hline 2 & Radix-2 $2^{4}$ MDF $[19,20]$ & $\frac{1}{2} \log _{2} N-2$ & $2 \log _{2} N$ & $\frac{3 N}{2}-2$ & $\mathrm{R}$ & $2 \mathrm{R}$ \\
\hline 2 & This work & $\frac{2}{3} \log _{2} N-\frac{5}{3}$ & $\frac{2}{3} \log _{2}\left(\frac{N}{2}\right)$ & $\frac{3 N}{2}-2$ & $\mathrm{R}$ & $2 \mathrm{R}$ \\
\hline 1 & Radix-2 $2^{3}$ SDF-4R [21] & $\frac{1}{3} \log _{2} N-1$ & $\frac{1}{3} \log _{2} N$ & $4(N-1)$ & $4 \mathrm{R}$ & $4 \mathrm{R}$ \\
\hline 4 & Radix-4 MDC & $\frac{3}{2} \log _{2} N-3$ & - & $\frac{5 N}{2}-4$ & $\mathrm{R}$ & $4 \mathrm{R}$ \\
\hline 4 & Radix-2 $2^{3}$ MDF [3] & $\frac{4}{3} \log _{2} N-4$ & $\frac{4}{3} \log _{2} N$ & $\frac{5 N}{2}-4$ & $\mathrm{R}$ & $4 \mathrm{R}$ \\
\hline 4 & Radix-2 $\cdot 4 \cdot 2$ MDC $[22]$ & $\log _{2} N-4$ & $\frac{9}{4} \log _{2} N$ & $\frac{5 N}{2}-4$ & $\mathrm{R}$ & $4 \mathrm{R}$ \\
\hline 4 & Radix-2 $2^{4}$ MDF [23] & $\log _{2} N-4$ & $4 \log _{2} N$ & $\frac{5 N}{2}-4$ & $\mathrm{R}$ & $4 \mathrm{R}$ \\
\hline 4 & This work & $\frac{4}{3} \log _{2} N-\frac{11}{3}$ & $\frac{4}{3} \log _{2} N-\frac{2}{3}$ & $\frac{5 N}{2}-4$ & $\mathrm{R}$ & $4 \mathrm{R}$ \\
\hline
\end{tabular}

higher sampling frequency is used in advanced systems. With our proposed architecture, the advantage is twofold. On one hand, the same control flow as the one needed for generation of multiple-channel FFT processors can be shared. On the other hand, we still exploit the radix- $2^{3}$ algorithm in hardware reduction. From the table, it is clear that our architecture is flexible and hardware efficient.

Basic arithmetic processing elements (PEs) are shown in Figure 2 for constructing various FFT processors. While PE1, PE2, and PE3 are used in the SDF architecture, PE4, PE5, and PE6 are instantiated in case parallel processing is needed. PE3 and PE6 compute the radix-2 butterfly operation. PE1 and PE4 handle the extra complex multiplication of $-j$. PE2 and PE5 deal with the trivial multiplications of $W_{8}^{1}$ as well as $W_{8}^{3}$ by shifters and adders. PE4 and PE5 are only utilized when the degree of parallelism is four. The delay buffer with a size greater than 16 is made up of a memory array addressing by an incrementer whose current value and previous value are adopted as the read and write addresses to guarantee the read operation done before the write operation at the same address.

The variable FFT sizes are achieved by the alternative data paths controlled by the multiplexers as shown in Figure 3, which is an example of 64-point to 4096-point variable-size single-channel FFT processor. For $N=2^{K}$, there are total $K$ stages. To perform the $2^{3 n}$-point FFT operation, where $3 n \leq K$, the signal directly enters the PE1 at the $(K-3 n+1)$ th stage. When $2 \cdot 2^{3 n}$-point FFT is desired, the signal feeds directly to PE3 at stage $(K-3 n)$. If $\left(2^{2} \cdot 2^{3 n}\right)$-point FFT is executed, we will route the signal going through PE1 at stage $(K-3(n+1)+1)$, bypassing the next PE2 and entering into PE3 and its successive stages. Meanwhile, the delay buffer of 

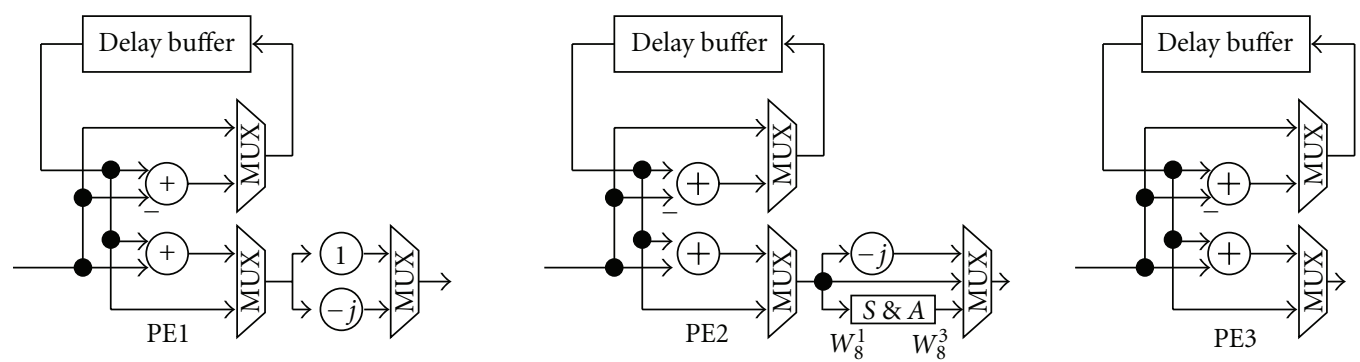

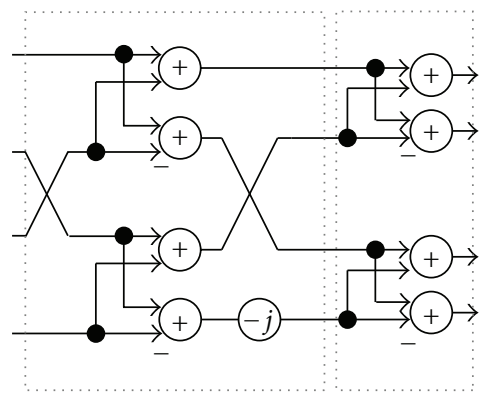

PE4

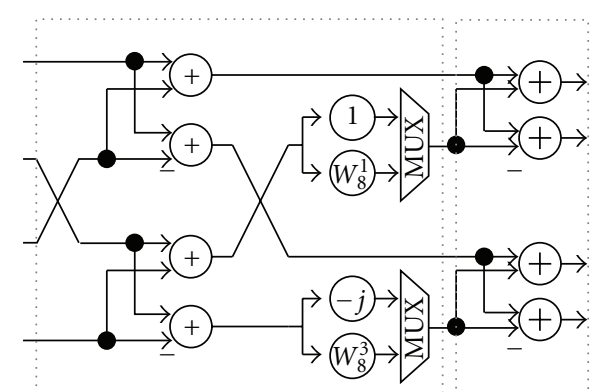

PE5

FIgURE 2: Block diagram of basic arithmetic processing elements.

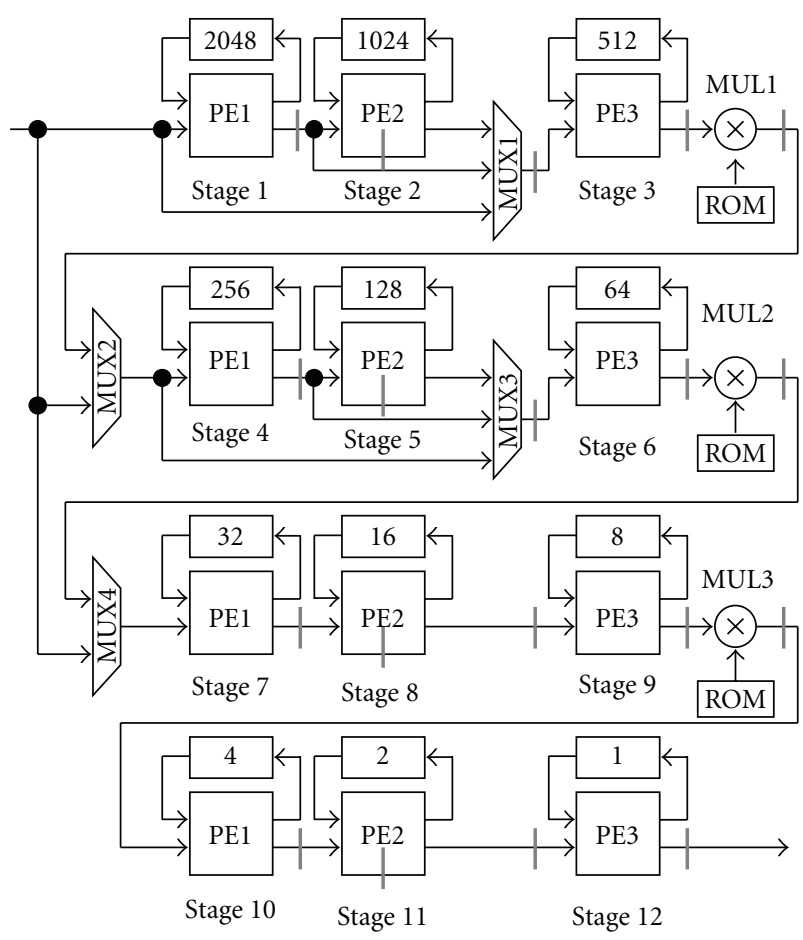

FIGURE 3: Architecture of the generated SISO variable-length radix- $2^{3}$ FFT processor.

PE1 will be programmed to use only one half of its original size, which can be done by simply using the arithmetic shift of the counter output to the left by 1 bit without changing the memory array. The gray vertical lines along the data path denote the possible pipeline-register insertion positions. If the required operating frequency is not high, then according to the information in the timing library, only parts of these pipeline registers are instantiated. On the contrary, all of them will exist if the clock frequency needs to be raised to over $100 \mathrm{MHz}$.

As to automatic generation of multichannel FFT IP, it basically can be regarded as constructing a two-dimensional $\mathrm{PE}$ array. The number of columns in the PE array relates to the number of stages. On the other hand, the number 


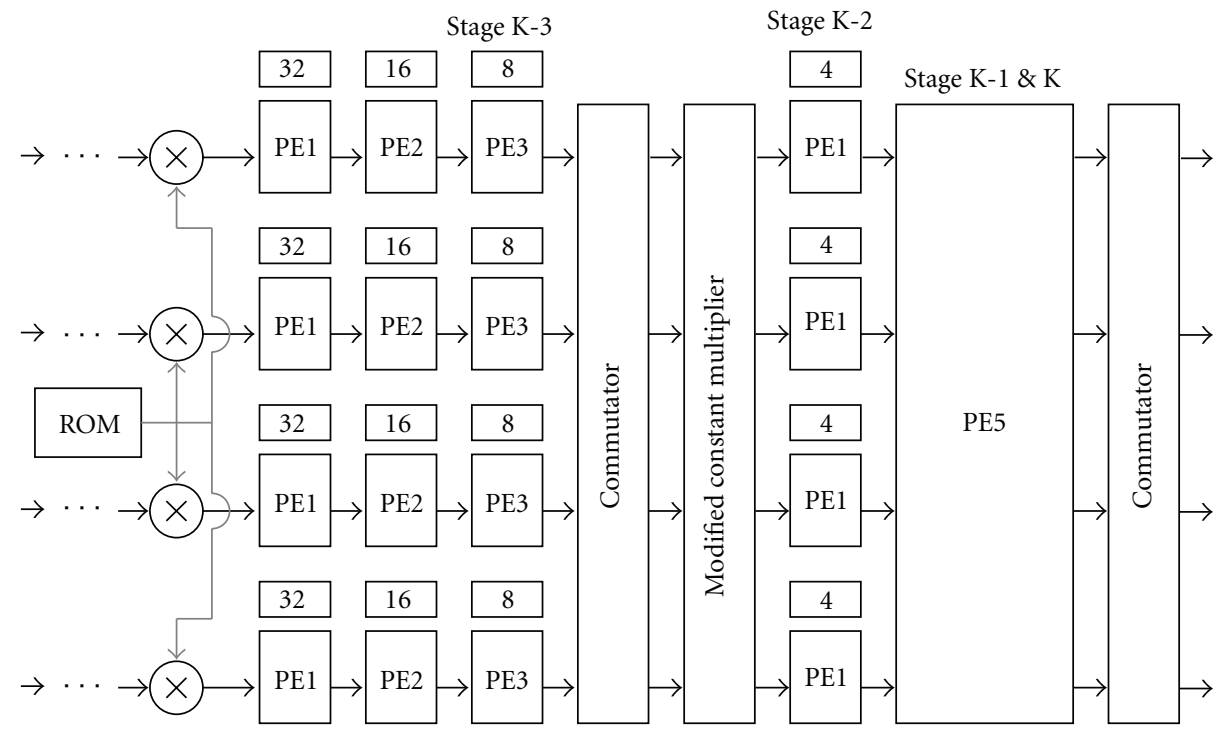

FIgURE 4: Architecture of a MIMO FFT processor with 4 channels.

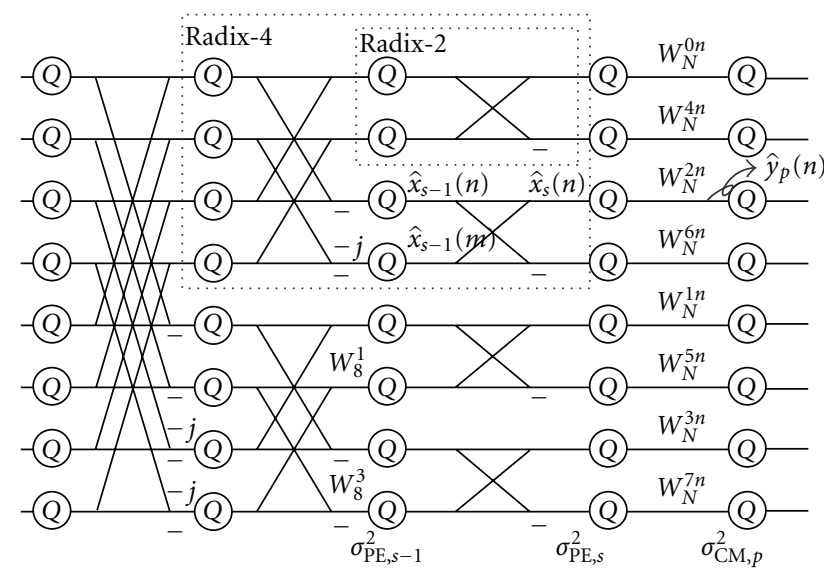

FIGURE 5: Signal flow graph of the radix- $2^{3}$ algorithm.

of rows corresponds to the number of channels. However, if we simply duplicate the single-channel FFT processor several times to obtain a multichannel FFT processor, the hardware redundancy exists. Therefore, the hardware sharing techniques are employed in the generated IP core. Generally, in $M$-channel FFT processors, because independent $M$ data streams are processed simultaneously, only one ROM table will be generated and its output is connected to $M$ twiddlefactor multipliers. The ROM table saves only twiddle factors in $[0, \pi / 4]$, and we use the symmetry of sine/cosine waveforms to derive the values of the remaining twiddle factors. In the special case of a four-channel FFT processor in MIMO systems, a modified constant multiplication module and PE5 are adopted to save hardware complexity in the tail stages as shown in Figure 4 [3]. The modified constant multiplication module contains eight sets of shifters and adders for the twiddle factors $W_{64}^{n}, n=1,2, \ldots, 8$, which can have $38 \%$ complexity reduction compared to four complex multipliers according to [3]. An extra commutator is required to reorder the four-channel signals so that different sets of shifters and adders can be used by the four data paths without conflict. As a result, for 4-channel FFT handling more than 64 points, architecture in Figure 4 is employed. If an FFT processor dealing with more than 256 points with parallelism level of 4 is required, architectures of Figures 1 and 4 will be combined and generated.

By adopting the radix $-2^{3}$ algorithm and the flexible architecture that utilizes both SDF and MDC, the proposed IP generator thus supports multichannel as well as parallel processing, one fixed-size or multiple variable-size, and userspecified operating frequency with reduced complexity.

\section{Finite Precision Effect and Word-Length Optimization}

To design a proper word-length searching procedure, we need to realize the mean squared quantization error due to the finite precision effect. Observe the signal flow graph of the radix- $2^{3}$ FFT operation as given in Figure 5 . It is clear that only two types of arithmetic computations are involved, that is, complex addition/subtraction and complex multiplication. In addition, the twiddle factors are all pure fractional numbers except for \pm 1 and 0 . Obviously they cause a long word length in the fractional part after multiplication. Hence, to avoid rapid growth in hardware complexity, truncation is necessary. In Figure 5, the circle with " $Q$ " denotes the introduction of the probable quantization effect due to truncation. In the following, the mean squared quantization errors resulted from these two types of arithmetic operations and the truncation are analyzed. Note that these analyses are also applicable to radix- 2 and radix- 4 algorithms.

3.1. Quantization Error after Complex Addition/Subtraction. Assume that two input signals to be summed are denoted as 
$\hat{x}_{s}(n)$ as well as $\hat{x}_{s}(m)$, where $x_{s}(n)$ is the $n$th signal at the sth stage, the notation $(\hat{*})$ indicates the quantized version of the signal, and $m=n+N / 2^{s}$. The output after complex addition/subtraction is given by

$$
\begin{aligned}
\hat{x}_{s+1}(n)= & \hat{x}_{r, s+1}(n)+j \hat{x}_{i, s+1}(n) \\
= & \left(x_{r, s+1}(n)+\delta_{r, s+1}(n)\right)+j\left(x_{i, s+1}(n)+\delta_{i, s+1}(n)\right) \\
= & \left(x_{r, s}(n)+x_{r, s}(m)+\delta_{r, s}(n)+\delta_{r, s}(m)\right) \\
& +j\left(x_{i, s}(n)+x_{i, s}(m)+\delta_{i, s}(n)+\delta_{i, s}(m)\right), \\
\hat{x}_{s+1}(m)= & \left(x_{r, s}(n)-x_{r, s}(m)+\delta_{r, s}(n)-\delta_{r, s}(m)\right) \\
& +j\left(x_{i, s}(n)-x_{i, s}(m)+\delta_{i, s}(n)-\delta_{i, s}(m)\right),
\end{aligned}
$$

where $x_{r, s}(n)$ and $x_{i, s}(n)$ denote the real part and imaginary part of $x_{s}(n)$ and $\delta_{r, s}(n)$ and $\delta_{i, s}(n)$ represent the real part and the imaginary part of the quantization error, which may have nonzero mean. Assume the mean square error at the sth PE stage due to $\delta_{r, s}(n)$ and $\delta_{i, s}(n)$ as $\sigma_{\mathrm{PE}, s}^{2}$. Note that one half of the signals at the $(s+1)$ th stage is computed by addition while the other half is computed by subtraction. Therefore, the mean of the quantization error $\left(\hat{x}_{s+1}(n)-x_{s+1}(n)\right)$ with $n=0,1, \ldots, N-1$ at stage $(s+1)$ is given by

$$
\mu_{\mathrm{PE}, s+1}=E\left\{\delta_{r, s}(n)\right\}+j E\left\{\delta_{i, s}(n)\right\}=\mu_{\mathrm{PE}, s} .
$$

The mean squared quantization error after addition and subtraction can be calculated respectively as

$$
\begin{gathered}
E\left\{\left|\left[\hat{x}_{r, s+1}(n)-x_{r, s+1}(n)\right]+j\left[\hat{x}_{i, s+1}(n)-x_{i, s+1}(n)\right]\right|^{2}\right\} \\
=E\left\{\left|\delta_{r, s}(n)+\delta_{r, s}(m)\right|^{2}\right\}+E\left\{\left|\delta_{i, s}(n)+\delta_{i, s}(m)\right|^{2}\right\}, \\
E\left\{\left|\left[\hat{x}_{r, s+1}(m)-x_{r, s+1}(m)\right]+j\left[\hat{x}_{i, s+1}(m)-x_{i, s+1}(m)\right]\right|^{2}\right\} \\
=E\left\{\left|\delta_{r, s}(n)-\delta_{r, s}(m)\right|^{2}\right\}+E\left\{\left|\delta_{i, s}(n)-\delta_{i, s}(m)\right|^{2}\right\} .
\end{gathered}
$$

With the assumption of uncorrelated quantization errors, the mean squared error at stage $(s+1)$ becomes

$$
\sigma_{\mathrm{PE}, s+1}^{2}=2 \sigma_{\mathrm{PE}, s}^{2}
$$

Details are shown in Appendix A.

3.2. Quantization Error after Complex Multiplication. Assume that $W_{r, p}(m)$ and $W_{i, p}(m)$ indicate the real part and the imaginary part of the $m$ th twiddle factor at the $p$ th complex multiplication block. The $n$th quantized signal $\hat{y}_{p}(n)$ after the $p$ th complex multiplication takes the form of

$$
\begin{aligned}
\hat{y}_{p}(n)= & \hat{y}_{r, p}(n)+j \hat{y}_{i, p}(n) \\
= & {\left[\left(x_{r, s}(n)+\delta_{r, s}(n)\right)+j\left(x_{i, s}(n)+\delta_{i, s}(n)\right)\right] } \\
& \cdot\left[\left(W_{r, p}(m)+\epsilon_{r, p}(m)\right)+j\left(W_{i, p}(m)+\epsilon_{i, p}(m)\right)\right] \\
\approx & {\left[\left(x_{r, s}(n) W_{r, p}(m)-x_{i, s}(n) W_{i, p}(m)\right)\right.} \\
& +\left(W_{r, p}(m) \delta_{r, s}(n)-W_{i, p}(m) \delta_{i, s}(n)\right) \\
& \left.+\left(x_{r, s}(n) \epsilon_{r, p}(m)-x_{i, s}(n) \epsilon_{i, p}(m)\right)\right] \\
+ & j\left[\left(x_{r, s}(n) W_{i, p}(m)+x_{i, s}(n) W_{r, p}(m)\right)\right. \\
& +\left(W_{i, p}(m) \delta_{r, s}(n)+W_{r, p}(m) \delta_{i, s}(n)\right) \\
& \left.+\left(x_{r, s}(n) \epsilon_{i, p}(m)+x_{i, s}(n) \epsilon_{r, p}(m)\right)\right]
\end{aligned}
$$

where $\epsilon_{r, p}(m)$ and $\epsilon_{i, p}(m)$ denote the real-part and the imaginary-part quantization errors of the twiddle factor. Since the twiddle factors can be predetermined by rounding operation, they can be assumed to have zero mean. The statistics of quantization errors after complex multiplication can be derived as

$$
\begin{gathered}
\mu_{\mathrm{CM}, p}=\left(E\left\{W_{r, p}(m)\right\} E\left\{\delta_{r, s}(n)\right\}-E\left\{W_{i, p}(m)\right\} E\left\{\delta_{i, s}(n)\right\}\right) \\
+j\left(E\left\{W_{i, p}(m)\right\} E\left\{\delta_{r, s}(n)\right\}+E\left\{W_{r, p}(m)\right\} E\left\{\delta_{i, s}(n)\right\}\right), \\
\sigma_{\mathrm{CM}, p}^{2} \approx E\left\{\mid\left[W_{r, p}(m) \delta_{r, s}(n)-W_{i, p}(m) \delta_{i, s}(n)\right.\right. \\
\left.\left.+x_{r, s}(n) \epsilon_{r, p}(m)-x_{i, s}(n) \epsilon_{i, p}(m)\right]\left.\right|^{2}\right\} \\
+E\left\{\mid\left[W_{i, p}(m) \delta_{r, s}(n)+W_{r, p}(m) \delta_{i, s}(n)\right.\right. \\
\left.\left.+x_{r, s}(n) \epsilon_{i, p}(m)+x_{i, s}(n) \epsilon_{r, p}(m)\right]\left.\right|^{2}\right\} .
\end{gathered}
$$

Similarly, by applying the assumption of uncorrelated errors of $\delta_{r, s}(n), \delta_{i, s}(n) \epsilon_{r, p}(m)$, and $\epsilon_{i, p}(m)$, and mutually independent random variables of the data paths and twiddle factors, the mean squared error becomes

$$
\begin{aligned}
\sigma_{\mathrm{CM}, p}^{2} \approx & E\left\{\left|W_{r, p}(m) \delta_{r, s}(n)\right|^{2}\right\}+E\left\{\left|W_{i, p}(m) \delta_{i, s}(n)\right|^{2}\right\} \\
& +E\left\{\left|x_{r, s}(n) \epsilon_{r, p}(m)\right|^{2}\right\}+E\left\{\left|x_{i, s}(n) \epsilon_{i, p}(m)\right|^{2}\right\} \\
& -2 E\left\{\left|W_{r, p}(m) W_{i, p}(m) \delta_{r, s}(n) \delta_{i, s}(n)\right|\right\} \\
& +E\left\{\left|W_{i, p}(m) \delta_{r, s}(n)\right|^{2}\right\}+E\left\{\left|W_{r, p}(m) \delta_{i, s}(n)\right|^{2}\right\}
\end{aligned}
$$




$$
\begin{aligned}
& +E\left\{\left|x_{r, s}(n) \epsilon_{i, p}(m)\right|^{2}\right\}+E\left\{\left|x_{i, s}(n) \epsilon_{r, p}(m)\right|^{2}\right\} \\
& +2 E\left\{\left|W_{r, p}(m) W_{i, p}(m) \delta_{r, s}(n) \delta_{i, s}(n)\right|\right\} \\
= & 2 E\left\{W_{r, p}^{2}(m)+W_{i, p}^{2}(m)\right\} \frac{\sigma_{\mathrm{PE}, s}^{2}}{2} \\
& +2 E\left\{x_{r, s}^{2}(n)+x_{i, s}^{2}(n)\right\} \frac{\sigma_{T, p}^{2}}{2},
\end{aligned}
$$

where the mean squared error of the twiddle factors at the $p$ th complex multiplication block, $E\left\{\epsilon_{r, p}^{2}+\epsilon_{i, p}^{2}\right\}$, is denoted as $\sigma_{T, p}^{2}$.

It is clear that in $(7)$, the term $\left(W_{r, p}^{2}(m)+W_{i, p}^{2}(m)\right)$ has unit magnitude. On the other hand, according to Parseval's theorem, we can derive the average of $\left(x_{r, s}^{2}(n)+x_{i, s}^{2}(n)\right)$. The derivations are available in Appendix B. In our case of the radix-2 butterfly,

$$
E\left\{x_{r, s}^{2}(n)+x_{i, s}^{2}(n)\right\}=\frac{2^{s}}{N^{2}} \sum_{k=0}^{N-1}|X(k)|^{2} .
$$

Generally, in OFDM systems, the frequency-domain data $X(k)$ are selected from some pre-determined constellations with normalized energy. Consequently, the averaged energy of frequency domain signal $X(k)$ can be easily computed. Thus, from (7) and (8), the mean squared error after complex multiplication becomes

$$
\sigma_{\mathrm{CM}, p}^{2} \approx \sigma_{\mathrm{PE}, s}^{2}+\frac{2^{s}}{N^{2}} \sum_{k=0}^{N-1}|X(k)|^{2} \sigma_{T, p}^{2}=\sigma_{\mathrm{PE}, s}^{2}+\frac{2^{s}}{N} \sigma_{T, p}^{2} .
$$

3.3. Quantization Error after Truncation. Two types of signal truncation are discussed here. One is truncation after multiplication and the other is truncation after addition/subtraction. Different error distributions can be observed in each case.

If the fractional parts of the twiddle factor and the data path contain $b_{t}$ and $b_{d}$ bits, respectively, then after complex multiplication, the word-length in the fractional part of the product becomes $b_{t}+b_{d}$ bits. Therefore, truncation is often performed. As shown in Figure 6, define $d=2^{-\left(b_{t}+b_{d}\right)}$ as the finest granularity. After truncation, we use $b_{m}$ bits in the fractional part. Note that $D=2^{-b_{m}}=2^{M} d$, where $M=$ $b_{t}+b_{d}-b_{m}$. Because FFT involves a lot of butterfly operations, according to the central limit theorem, for $d \ll D$, the quantization error can be modeled as Gaussian distribution which may be biased and thus have a nonzero mean $\alpha$ as indicated in Figure 6. The distance between the floatingpoint representation $y_{r, p}(n)$ and the nearest fixed-point representation in the finest granularity is denoted by $t$. After truncation, all the signals $\hat{y}_{r, p}(n)$ inside one of the shadowed region now are classified as $\hat{z}_{r, p}(n)$ and have squared error of $(t+i d-l D)^{2}$, where $l=0, \pm 1, \pm 2, \ldots i$ has equal probability ranging from 0 to $2^{M}-1$ and $t$ is assumed to be uniformly distributed in $[0, d)$.
Define the conditional probability on $t$ and $i$ of the quantization error falling in each shadowed region indexed by $l$ as $g(l \mid t, i)$, which can be computed as

$$
\begin{aligned}
g(l \mid t, i) & =\int_{-t-i d+l D}^{-t-i d+(l+1) D} \frac{1}{\sqrt{2 \pi} \nu} e^{-(x+\alpha)^{2} / 2 v^{2}} d x \\
& =q\left(\frac{-t-i d+\alpha+l D}{\nu}\right)-q\left(\frac{-t-i d+\alpha+(l+1) D}{\nu}\right),
\end{aligned}
$$

where

$$
q(y)=\int_{y}^{\infty} \frac{1}{\sqrt{2 \pi}} e^{-x^{2}} d x,
$$

and $v^{2}$ is the variance of quantization error in either the real or the imaginary part after complex multiplication and before truncation, which can be calculated as $(1 / 2)\left(\sigma_{\mathrm{CM}, p}^{2}-\right.$ $\left.\mu_{\mathrm{CM}, p}^{2}\right)$. Denote $f_{T}(t)$ as the probability density function of $t$ and $f_{T}(t)=1 / d$. Then, after truncation of the bits in the fractional part, the mean squared error of the complex output becomes

$$
\sigma_{T 1, p}^{2}=2 \sum_{l=-\infty}^{\infty} \sum_{i=0}^{2^{M}-1} \frac{1}{2^{M}} \int_{0}^{d} g(l \mid t, i) f_{T}(t)(t+i d-l D)^{2} d t,
$$

and the mean of quantization error after truncation can be given by

$\mu_{T 1, p}=(1+j) \sum_{l=-\infty}^{\infty} \sum_{i=0}^{2^{M}-1} \frac{1}{2^{M}} \int_{0}^{d} g(l \mid t, i) f_{T}(t)(t+i d-l D) d t$.

Equations (12) and (13), which can be computed by numeric approaches, play an important role to analyze the statistics of quantization errors owing to truncation after complex multiplications.

For those cases which use one-bit truncation after the butterfly operation, the assumption of Gaussian distribution is not suitable because the inequality $D \gg d$ is not satisfied. We then utilize the same assumption of uniform distribution as in [25]. Thus, one half of the signal remains the same, and the other half has additional quantization error of $d$. The mean of quantization error after LSB truncation can be calculated as

$$
\begin{aligned}
\mu_{T 2, s}= & {\left[\frac{1}{2} E\left\{\delta_{r, s}(n)\right\}+\frac{1}{2} E\left\{\delta_{r, s}(n)+d\right\}\right] } \\
& +j\left[\frac{1}{2} E\left\{\delta_{i, s}(n)\right\}+\frac{1}{2} E\left\{\delta_{i, s}(n)+d\right\}\right]=\mu_{\mathrm{PE}, s}+d \frac{1+j}{2} .
\end{aligned}
$$

The mean squared error after LSB truncation can be derived as

$$
\begin{aligned}
\sigma_{T 2, s}^{2}= & \frac{1}{2} E\left\{\delta_{r, s}^{2}(n)\right\}+\frac{1}{2} E\left\{\left(\delta_{r, s}(n)+d\right)^{2}\right\} \\
& +\frac{1}{2} E\left\{\delta_{i, s}^{2}(n)\right\}+\frac{1}{2} E\left\{\left(\delta_{i, s}(n)+d\right)^{2}\right\} \\
= & \sigma_{\mathrm{PE}, s}^{2}+d E\left\{\delta_{r, s}(n)+\delta_{i, s}(n)\right\}+d^{2} .
\end{aligned}
$$


Unlike in [25], we introduce an extra term to account for the possible nonzero mean after truncation. In the following, we can see its influence on the accuracy of the analytic mean square quantization errors.

3.4. Discussion on Word-Length Optimization. According to the previous analyses for the finite precision effect in an FFT processor, some observations are summarized below.

(i) In the radix $-2^{3}$ single-path delay feedback architecture, the average signal energy is increased by 2 according to Parseval's theorem (see Appendix B), while the mean squared quantization error also doubles after butterfly operation as given by (4). Define a signal-to-quantization error ratio (SQNR) as

$$
\mathrm{SQNR}=10 \cdot \log _{10} \frac{E\left\{\left|x_{s}(n)\right|^{2}\right\}}{\sigma_{\mathrm{PE}, s}^{2}} .
$$

Hence, if the signal is not truncated after butterfly operation, the SQNR remains the same.

(ii) The SQNR decreases after complex multiplication because of the finite precision of twiddle factors. The quantization errors in twiddle factors are further scaled by the average energy of the signal to be multiplied as indicated in (9). Consequently, the word-length settings of twiddle factors and data paths should be decided individually. Moreover, (9), also reveals the reason that a shorter word-length can always be assigned for twiddle factors than the data path in an FFT processor since $2^{s} / N \ll 1$.

(iii) The mean squared quantization errors increase monotonically from the first stage to the last stage. For those stages at which quantization errors accumulate and severely pollute the least significant bits (LSBs) of finite-precision signals, proper truncation introduces only negligible degradation compared to $\sigma_{\mathrm{PE}, s}^{2}$ as in (15) for $d^{2} \ll \sigma_{\mathrm{PE}, s}^{2}$.

To verify the previous analysis, the analytic results (12) and simulated results are compared in Figure 7 . The horizontal axis represents the word-length $b_{m}$ while the vertical axis denotes the MSE. Twiddle factor multiplications for 64point and 512-point FFT operations are both evaluated. In both cases, the twiddle factors are quantized to 10 bits in their fractional part. The fractional part of the input data-path signal before multiplication is represented by 11 bits and 12 bits in 64-point and 512-point FFT, respectively. Accordingly, without truncation, the fractional parts become 21 and 22 bits. From the figure, we can see that the analytic results approach the simulated results. Besides, the proper wordlength $b_{m}$ can be selected around the knee point close to the error floor, which implies that only slight degradation occurs.

In Figure 8, the analytic results by using (4), (9), (12), and (15) and the simulated results of the mean squared quantization errors at each stage for 512-point FFT are compared. In addition, we also provide the curve of the analytic results by [25]. The effect of $W_{8}^{1}$ and $W_{8}^{3}$ in PE2

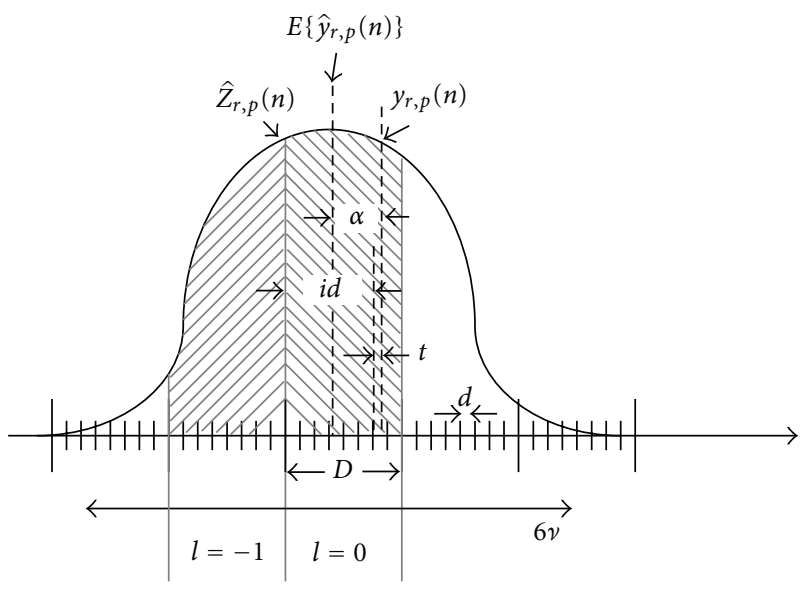

FIGURE 6: Quantization error distribution.

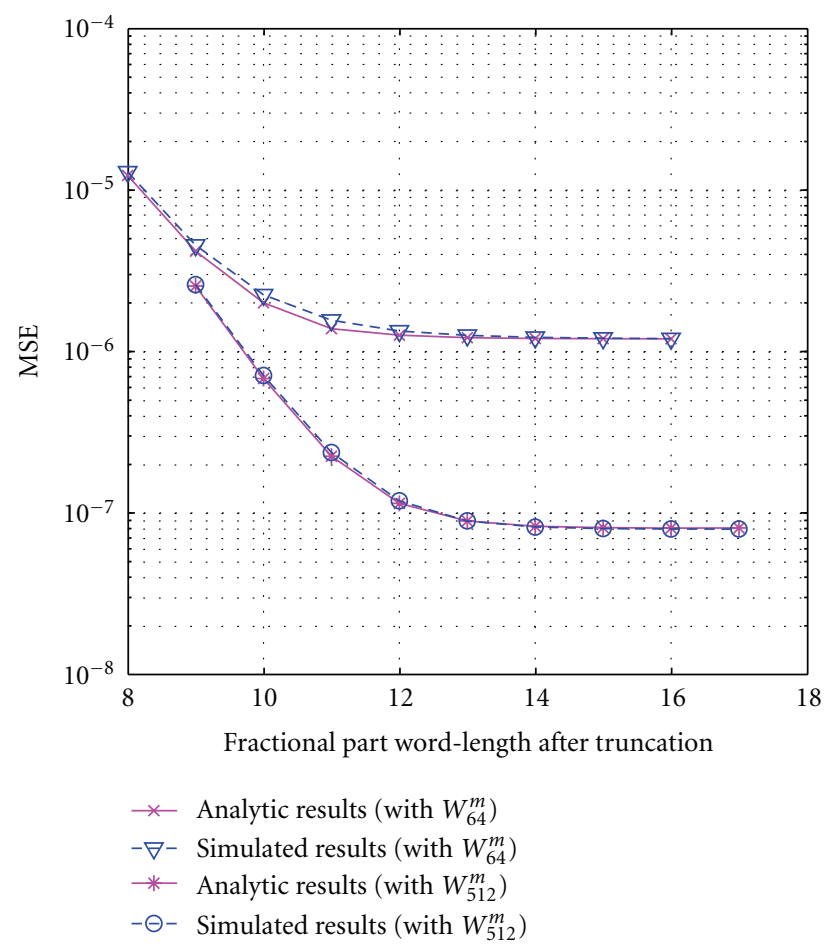

FIGURE 7: Analytic and simulated quantization mean squared error after truncation.

is ignored temporarily. The word lengths of the output at each stage after truncation are also indicated. It can be seen that if there is no truncation after the PE stages, the slope of the segment is $\log (2) /$ stage. If a proper word length around the knee point is chosen after complex multiplication, a nonzero slope of the segment appears but is still less than $\log (2) /$ stage. On the other hand, if truncation is performed after complex addition/subtraction, the slope becomes steep. This figure demonstrates that our analytic result that considers the bias effect after truncation and uses Gaussian distribution approximating the quantization error 


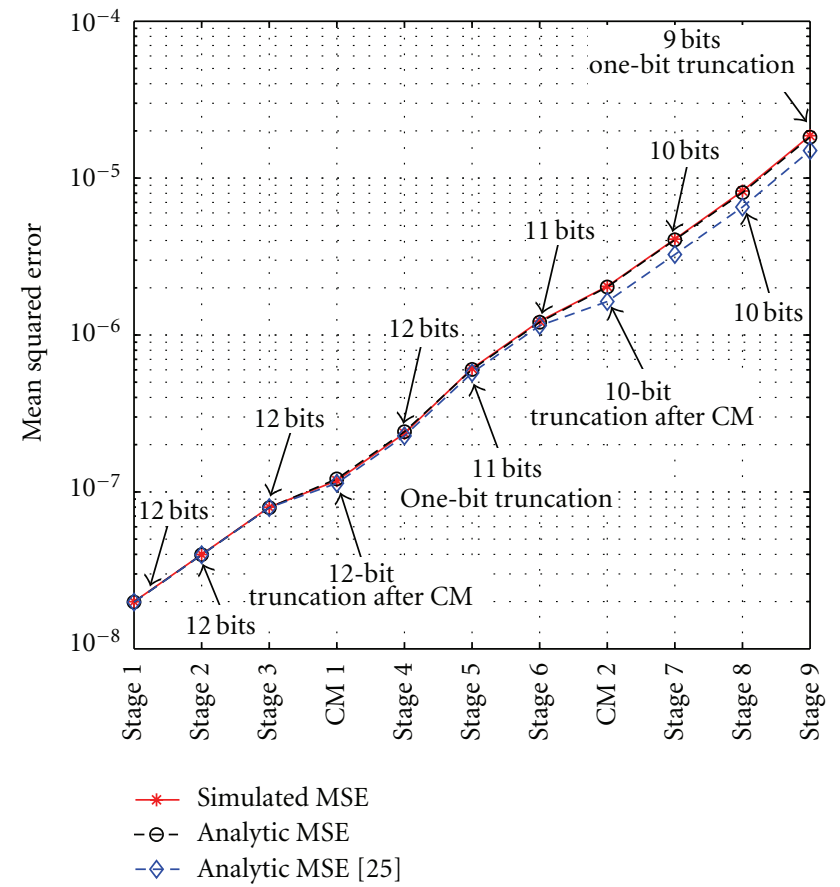

Figure 8: Comparison of analytic and simulated mean squared quantization errors at each stage in a 512-point FFT processor.

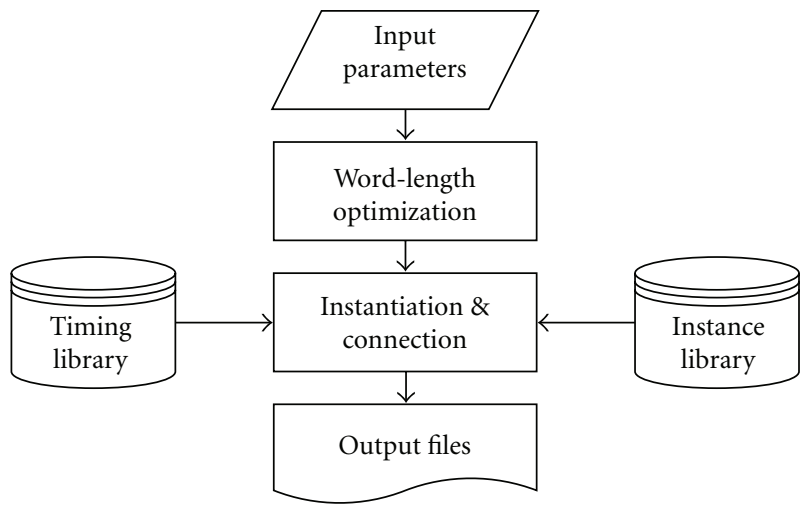

FIGURE 9: Flowchart of the proposed IP generator.

after complex multiplication can estimate the finite precision effect more accurately.

\section{Work Flow}

The work flow of the IP generator is indicated in Figure 9. In the first step, a user assigns his options such as the FFT size, configurations of parallelism, target operating frequency, allowable SQNR, and the FFT/IFFT mode for his desired IP core. Then, in order to minimize the finite precision effect, the word-length of each block will be optimized based on the SQNR criterion. In the third step, the IP generator instantiates the related submodules from the library and connects those submodules in the highesthierarchy top module. Finally, together with the desired hardware description language of the FFT processor, we also provide the test bench to users. We will describe the details of these four steps in the following.

4.1. Input Parameters. The proposed IP generator provides five main options.

4.1.1. FFT or IFFT Mode. In an OFDM system, the IFFT operation is needed in a transmitter while the FFT operation should be done in a receiver. The IFFT operation can be written as

$$
x(n)=\frac{1}{N} \sum_{k=0}^{N-1} X(k) W_{N}^{-n k}=\frac{1}{N}\left[\sum_{k=0}^{N-1} X^{*}(k) W_{N}^{n k}\right]^{*},
$$

which can be interpreted as applying the FFT operation to the complex conjugate of the inputs and then dividing the complex conjugate of the FFT output by $N$. Since $N$ is a power of two, no extra hardware is required for the division. Hence, the proposed IP generator can provide the IFFT processor by incorporating additional paths to derive the 2's complement of the imaginary part of both the inputs to the FFT processor and outputs from the FFT processor.

4.1.2. FFT/IFFT Size. In Table 1, we can see that the current and emerging OFDM standards mainly use FFT/IFFT sizes up to 8192 . Consequently, our IP generator can provide one single-size FFT/IFFT processor from 8 to 8192 points by cascading adequate processing elements and also produce a variable-size FFT/IFFT processor in the range of 64 to 4096 points by adding multiplexers to control the data paths.

4.1.3. Sampling Rate. The generated FFT/IFFT processor must fulfill the system requirement of real-time operation. The proposed IP generator automatically inserts the necessary pipeline registers in the positions as indicated by the gray vertical lines in Figure 3 to reduce the critical path delay and thus satisfies the target of working frequency. In the timing library, we have constructed a table listing the critical path delay of PEs and multipliers. The highest frequency around $140 \mathrm{MHz}$ is obtained in 90-nm FPGA, when the critical path contains only a complex multiplier.

4.1.4. SQNR Value. The finite-word-length representation of the FFT/IFFT processor inevitably introduces quantization errors, which degrade system performance. Therefore, the word lengths of the generated FFT/IFFT IP core must be optimized according to the requested SQNR value.

4.1.5. Multiple-Channel and Parallel Processing. The generated processor can support up to eight-channel FFT/IFFT operations to cover the needs in MIMO-OFDM systems. In addition, parallelism degrees of two or four to enhance throughputs are also implemented to support wide-band applications such as UWB.

4.2. Word-Length Optimization. Consider the hardware complexity related with the word-length settings. The 
TABLE 3: One example of the proposed fractional-part word-length search procedure.

\begin{tabular}{|c|c|c|c|c|c|c|c|c|c|c|c|c|c|}
\hline $\begin{array}{l}\text { Search } \\
\text { phase }\end{array}$ & Stage 1 & Stage 2 & CMul 1 & Stage 3 & Stage 4 & Stage 5 & CMul 2 & Stage 6 & Stage 7 & Stage 8 & Twiddle & $\begin{array}{c}\text { Simulated } \\
\text { SQNR }\end{array}$ & $\begin{array}{c}\text { Analytic } \\
\text { SQNR }\end{array}$ \\
\hline \multirow{8}{*}{1} & 11 & 11 & 11 & 11 & 11 & 11 & 11 & 11 & 11 & 11 & 11 & 47.06 & 46.51 \\
\hline & 12 & 12 & 12 & 12 & 12 & 12 & 12 & 12 & 12 & 12 & 12 & 53.07 & 52.53 \\
\hline & 13 & 13 & 13 & 13 & 13 & 13 & 13 & 13 & 13 & 13 & 13 & 59.08 & 58.55 \\
\hline & 13 & 13 & 13 & 13 & 13 & 13 & 13 & 13 & 13 & 13 & 12 & 59.03 & 58.51 \\
\hline & 13 & 13 & 13 & 13 & 13 & 13 & 13 & 13 & 13 & 13 & 11 & 58.91 & 58.38 \\
\hline & 13 & 13 & 13 & 13 & 13 & 13 & 13 & 13 & 13 & 13 & 10 & 58.52 & 58.03 \\
\hline & 13 & 13 & 13 & 13 & 13 & 13 & 13 & 13 & 13 & 13 & 9 & 56.86 & 56.49 \\
\hline & 13 & 13 & 13 & 13 & 13 & 13 & 13 & 13 & 13 & 13 & 8 & 53.58 & 53.43 \\
\hline \multirow{7}{*}{2} & 13 & 13 & 13 & 13 & 13 & 13 & 13 & 13 & 13 & 12 & 9 & 56.72 & 56.35 \\
\hline & 13 & 13 & 13 & 13 & 13 & 13 & 13 & 13 & 12 & 12 & 9 & 56.53 & 56.22 \\
\hline & 13 & 13 & 13 & 13 & 13 & 13 & 13 & 12 & 12 & 12 & 9 & 56.23 & 55.98 \\
\hline & 13 & 13 & 13 & 13 & 13 & 12 & 12 & 12 & 12 & 12 & 9 & 55.48 & 55.10 \\
\hline & 13 & 13 & 13 & 13 & 12 & 12 & 12 & 12 & 12 & 12 & 9 & 54.64 & 54.48 \\
\hline & 13 & 13 & 13 & 13 & 13 & 12 & 12 & 12 & 12 & 11 & 9 & 55.04 & 54.72 \\
\hline & 13 & 13 & 13 & 13 & 13 & 12 & 12 & 12 & 11 & 11 & 9 & 54.50 & 54.38 \\
\hline
\end{tabular}

smaller word length in processing elements, the less complexity the complex adder/subtractor and the delay buffer. If a smaller word length is assigned to twiddle factors, the size of ROM tables can be scaled down linearly and the size of the complex multiplier can also be reduced, which saves more in silicon cost. The proposed IP generator can automatically search for the optimal word-length setting of each stage, which is a feature that the conventional IP generators do not provide.

Exhaustive search for optimal word lengths is a timeconsuming work. Observing the pipeline architecture, if the data-path at earlier stages uses a smaller word length, the delay elements can save more and a smaller-size complex multiplier is probably instantiated. Hence, we proposed a procedure which includes two search phases, that is, global search and local search, which aim to use smaller wordlength settings at the earlier stages. Initially, the same word length of the fractional part is set at all the PE stages. In the first phase, that is, the global search, the fractional-part word lengths of all the PE stages are increased or decreased together until an SQNR value of the FFT output closest to but greater than the target value is obtained. Subsequently, the reduction of the twiddle-factor word length is not ceased until the SQNR value is below the target value. In fact, the global search phase only determines the finest precision of data paths and twiddle factors, which has also been proposed in [16]. On the other hand, it has been pointed out in [25] that using varying word lengths at each stage is viable when the request of the IP that is optimized for each specific application is eager. We then proposed a second phase to fine tune the word length at each stage. The quantization error accumulates and thus the LSBs may be contaminated by quantization errors. We then truncate the LSB from the last stage to examine if the target SQNR can be still fulfilled. If the answer is true, then the test of LSB truncation proceeds to the earlier stages sequentially until the SQNR value is not satisfied. When it happens, we then restore the truncation at that stage and initiate a new iteration of LSB truncation from the last stage again. The procedure goes on so that the word length at each stage can be minimized.

Table 3 gives the results of word-length optimization procedure in the global search phase and the local search phase for 256-point FFT with an SQNR requirement of $55 \mathrm{~dB}$. As mentioned earlier, in the global search phase, one fractional part word length of all the PEs and one fractional-part word length of all the twiddle factors are chosen, respectively. We can see that if the LSB at stage 4 is eliminated, the SQNR value becomes unsatisfying. 

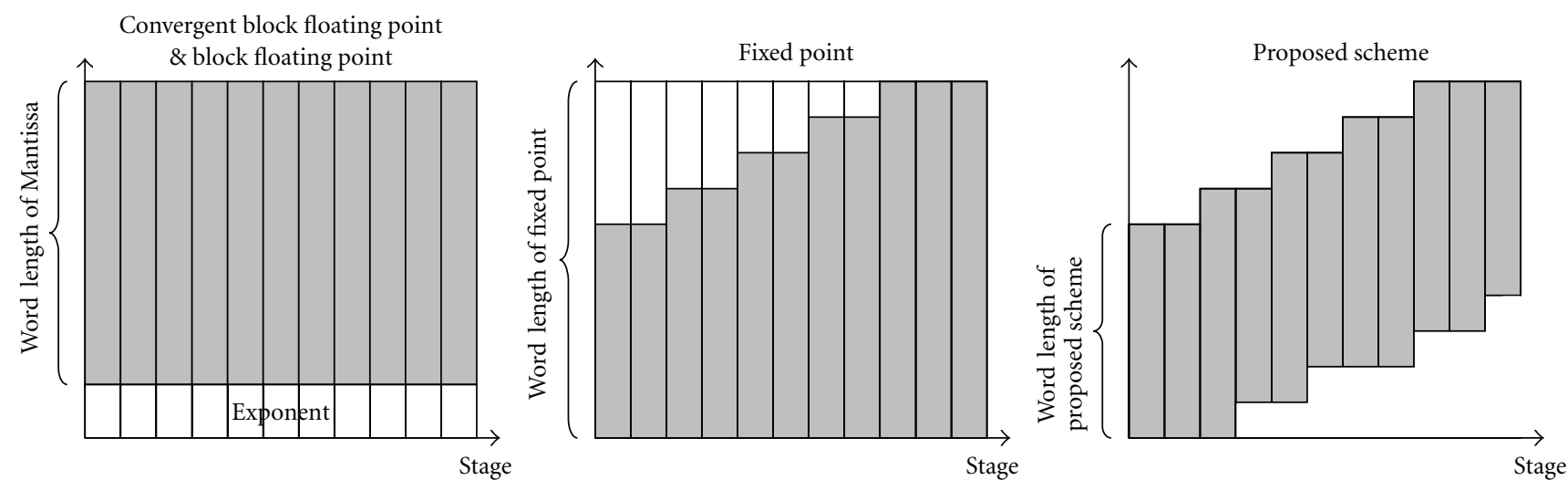

(a)

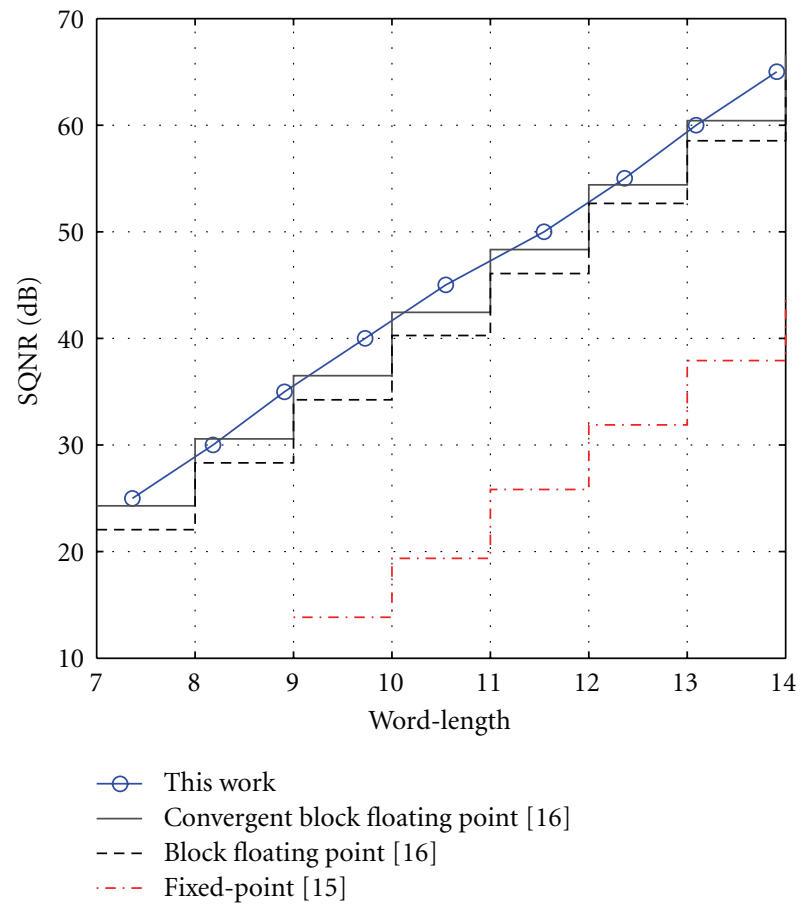

(b)

FIGURE 10: (a) Illustration of different data-path representations and (b) comparison of SQNR values versus word-length settings by different representations.

Therefore, we can only remove one LSB from stage 5 to stage 8 in the first iteration. Subsequently, the second iteration starts to truncate one LSB again from the last stage. This time, one LSB is removed only at stage 8 .

In Figure 10(a), we illustrate the word-length assignment of different representations at each stage, and in Figure 10(b), the SQNR values versus word lengths under different representations are given, where the word length of twiddle factor is set to 18 bits to get rid of its influence and the FFT size is 2048 points. The block-floating point representation uses an exponent at each stage to absorb the variation of dynamic ranges [16]. The convergent block-floating point representation has several scaling factors at one stage and one for each group [26]. It converges to one exponent for one FFT output. The fixed-point representation has only one format for all the stages, and thus it must take into consideration both the dynamic range and the precision simultaneously [15]. The effective range of each representation is indicated by the gray colors in Figure 10(a). With the block-floating point and convergent block-floating point representations, we can effectively use the entire dynamic range and thus definitely better performance can be achieved than the processor with the fixed-point representation, which may cause a waste in dynamic range at the early stages. However, in the conventional word-length searching algorithms for fixedpoint [15] and block-floating point [16] representations, 1 bit increase/decrease in the word length results in 6-dB change in SQNR. As a result, the processor must choose the word-length setting that produces SQNR value greater than the target value. With the proposed algorithm, we 
can approach the desired SQNR value by removing those harmless LSBs at the last stages compared to the fixed-point representation as shown in Figure 10(a) and can employ more flexible and adequate word lengths at each stage. Since the word lengths at each stage may be different, we depict the averaged word length of the proposed scheme in Figure 10(b). It is thus clear that our proposed word-length algorithm can meet the requirement of any user-specified SQNR value with reduced silicon cost.

4.3. Instantiation and Connection. Since the architecture to be generated is very regular, in the library we have prepared the basic submodules such as PE1 to PE6, a complex multiplier, a memory array, shift registers, pipeline registers, a commutator, and multiplexers. After the optimal wordlengths are derived, we can instantiate related submodules in the top module.

Nested FOR loops are used in the program to do the instantiation. In the outer loop, the program will judge which processing elements should be inserted, whether a multiplexer is required or not to control the signal flow, and whether a pipeline register should be included at the current stage. The look-up tables for twiddle factors will be automatically generated after its word length and the table size is determined. For the multichannel configuration, the second inner loop is used to duplicate the submodules that can not be shared. Once the instantiations of all the submodules are complete, the wires that connect these input and output ports are declared and constructed. Sign extension and LSB truncation are performed necessarily to ensure correct signal propagation between stages with different representation formats.

4.4. File Output and Test Bench. Finally, our IP generator will provide the user an FFT processor IP core and one test bench to facilitate its verification. For a variable-size FFT processor, the multiple test benches are offered to verify the correctness of each respective size.

\section{Experimental Results and Comparisons}

To verify the proposed IP generator, design examples of different configurations are tested as shown in Table 4. First, we use the IP generator to generate FFT IP cores. Their function has been examined to be correct with the automatically generated test bench. The desired SQNR as well as the resulted SQNR that adopts the simulated values in the search procedure is also available in the table. In Table 4(a), the performance and complexity of the FFT IP cores that are implemented by FPGA of device xc4vsx55-12 are given. The hardware complexity is evaluated in terms of FPGA resources of all design examples. The number of flip-flops is related with the pipeline registers and short delay buffers, while the number of slices reflects the logic complexity including distributed RAMs for long delay buffers and ROMs for twiddle-factor lookup tables. The DSP slices correspond to the multipliers. In our generated IP core, the DSP slices divided by four is exactly the number of complex multipliers.
For applications in UWB systems with a sampling rate of $528 \mathrm{MHz}$ and a throughput requirement more than 410 Mega samples, four parallel processing blocks are used so that the operating frequency can be reduced by a factor of 4 [3, $24]$. The throughput is calculated by the maximum operating frequency derived after synthesis. It is clear that the generated FFT IP meets the requirements of the UWB systems. For two-channel FFT processors, the complexity grows almost linearly. In addition, we can see the advantages of the modified constant multipliers in Figure 4 by comparing the implementation results of $802.11 \mathrm{n}$ with one channel and four channels. We can see that the DSP slices are reduced from $8 \times 4$ to 16 , a $50 \%$ reduction in complex multipliers. And the number of slice grows due to the complexity of shifters and adders in constant multipliers. As to the large-size FFT processors for 3GPP-LTE or DVB-T, the advantage of the radix $-2^{3}$ algorithm is clear in that it results in small increase of the number of complex multipliers. However, in these FFT processors, large ROM tables with 256 entries in 2048-point FFT and with 1024 entries in 8192-point FFT are required. Also, the long delay buffers implemented by distributed RAMs are also entailed. Both occupy large resources of the number of slices. Although block RAMs in FPGA can be used instead, owing to that the RAM macro is vendor specific, we still use the memory array, thus being implemented by distributed RAMs, to support the applications of the generated IP cores in cell-base design flow. On the righthand side of the table, we compare the IPs generated by different generators. Due to the fact that the pipeline registers are not inserted arbitrarily, equal throughput of the generated FFT processors is not straightforward to come by. However, we can normalize the hardware complexity to the throughput and evaluate the relative complexity as the ratio indicated in the parenthesis. With the radix $-2^{3}$ algorithm and the flexible architecture, our generated IP core uses less flip-flops and DSP slices (complex multipliers) with a slightly increased number of slices compared to the ones generated by Xilinx Logicore and the Spiral program. Thus, the hardware efficiency is better.

In Table 4(b), the synthesis results of several generated FFT IP cores by Design Compiler in $90 \mathrm{~nm}$ UMC CMOS technology are listed. The maximum frequency is derived by the critical path delay of typical cell library under 1-V supply voltage. Since the word length at each stage varies in our works, the average word length of all the butterfly stages is shown. To get further insight into their logic components, we indicate the equivalent gate counts of combinational logic and noncombinational logic. The normalized area is provided for fair comparison. The original area in their respective CMOS technology is also given in the parenthesis. The throughput is derived based on the maximum operating clock frequency. The power consumption is estimated from the synthesized results at $1-V$ supply voltage. Usually it is pessimistic compared to the measurement results. Other designs of 64-point FFT processors for 802.11a, 256-point FFT processors for $802.16 \mathrm{e}$, and an 8-channel FFT processor are also included. Because the timing information of the generator is mainly derived through the results in Virtex-4 FPGA, for the cell-based design flow, we push the maximum 
TABLE 4: Experimental results and comparisons of various generated FFT/IFFT processors implemented (a) by FPGA and (b) by cell-base design flow.

(a)

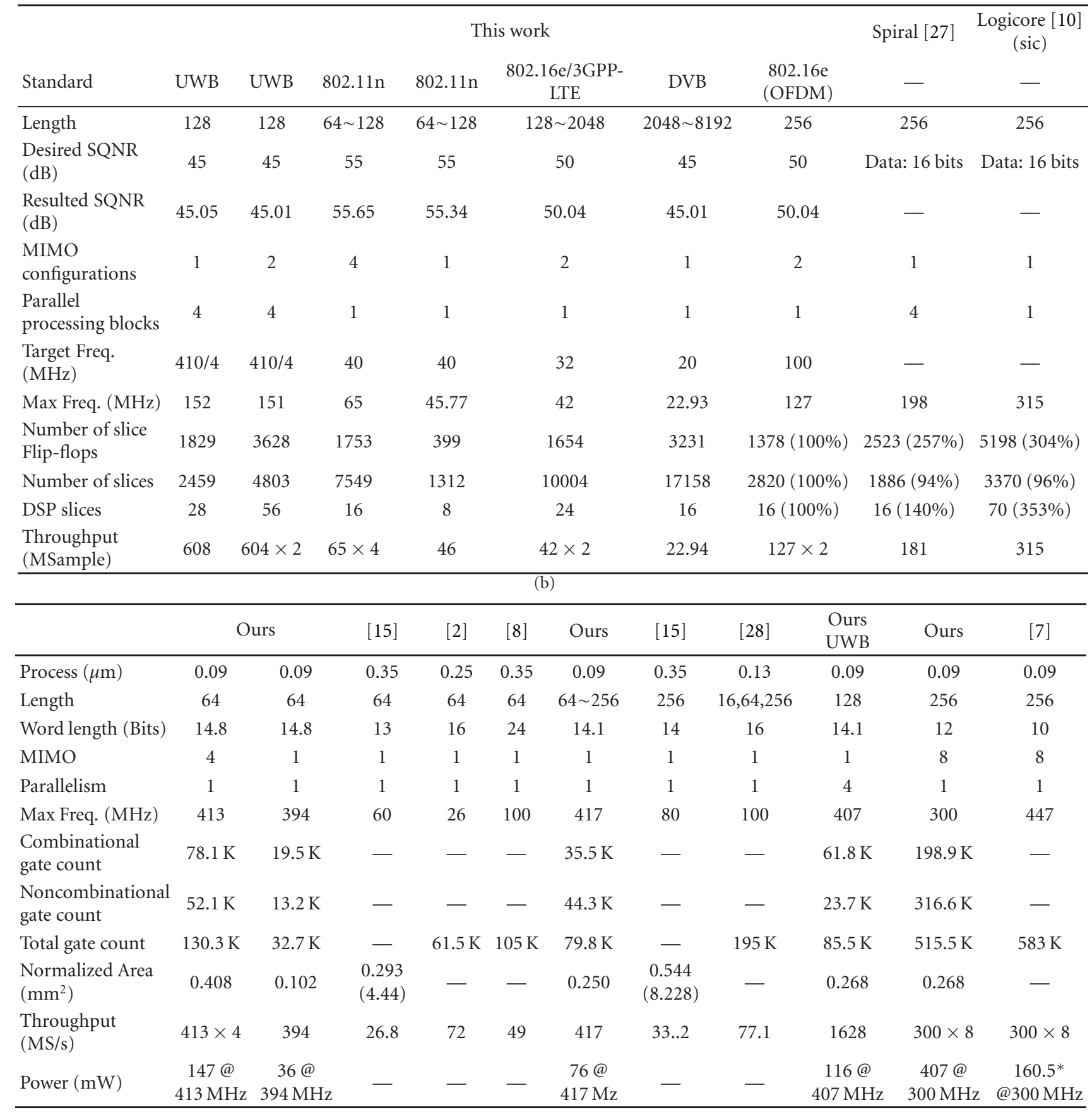

* measurement.

operating clock frequency of our works to the limit. As to the 256-point FFT processor, we can see that the ratio of noncombinational logic gates to total gates increases due to the delay elements, whose quantity is proportional to the FFT size. With the parallel processing architecture, we can support FFT processors for Giga samples per second in advanced communication systems. For all the cases, our IP generator can generate FFT processors with aggressive throughput and efficient hardware compared to other generators. From the table, it is shown that this IP generator is more competitive to generate FFT processors for various OFDM systems than previous works.

Although we do not address issues for power reduction, such as dynamic voltage and frequency scaling in [7] and thus the power consumption seems larger, the system-level power saving techniques can still be applied to obtain a 
low-power FFT IP by appropriate parameter settings. For example, we can set higher operating clock frequency than the nominal system sampling frequency to generate the IP that has short critical path delay and then scale down the supply voltage or synthesize it with a low-speed lowleakage library [29]. However, there are also some limitations that the proposed IP generator can not fully replace the manually designed application-specific IC (ASIC), like the use of sleep transistors or multiple-threshold transistors, especially in nanotechnology. Besides, in large-size FFT processors, instead of shift registers, the delay buffers are usually implemented by SRAMs, which are vendor specific and are not built-in. However, with the proposed automatic generator, the majority of the design efforts are saved.

\section{Conclusion}

To reduce the hardware design efforts spent on different FFT/IFFT processors for several communication standards and systems, an IP generator is developed. The proposed generator uses the higher radix algorithm and thus can save the number of complex multipliers in the generated FFT IP cores. In addition, we analyze the finite precision effect of the radix-2/4/8 algorithm, and a more accurate analytic result is derived. By observing the properties of the finite precision effect in FFT operation, an effective word-length searching procedure is proposed. With word-length optimization, a good tradeoff can be selected between complexity and accuracy. Besides, the pipelined architecture facilitates cutting off critical paths, and hence the generated FFT IP cores can be driven by a suitable clock frequency specified by users to introduce appropriate pipeline registers. The configurations of the variable-size and multichannel modes fulfill the needs of prosperous communication standards. To meet the throughput requirements, parallel processing is also incorporated. In summary, the proposed IP generator offers more flexibility and configurability than conventional solutions for recent MIMO-OFDM systems. Experimental results have demonstrated its capability and feasibility to generate a hardware-efficient design.

\section{Appendices}

\section{A. Derivation of Mean Square Error after Butterfly Operation}

The mean squared error of the signal at stage $(s+1)$ after complex addition takes the form of

$$
\begin{aligned}
& \sigma_{s+1, \text { add }}^{2} \\
&= E\left\{\left|\left[\hat{x}_{r, s+1}(n)-x_{r, s+1}(n)\right]+j\left[\hat{x}_{i, s+1}(n)-x_{i, s+1}(n)\right]\right|^{2}\right\} \\
&= E\left\{\left|\left[\delta_{r, s}(n)+\delta_{r, s}(m)\right]+j\left[\delta_{i, s}(n)+\delta_{i, s}(m)\right]\right|^{2}\right\} \\
&= E\left\{\left|\delta_{r, s}(n)+\delta_{r, s}(m)\right|^{2}\right\}+E\left\{\left|\delta_{i, s}(n)+\delta_{i, s}(m)\right|^{2}\right\} \\
&= E\left\{\delta_{r, s}^{2}(n)\right\}+E\left\{\delta_{r, s}^{2}(m)\right\}+2 E\left\{\delta_{r, s}(n)\right\} E\left\{\delta_{r, s}(m)\right\} \\
&+E\left\{\delta_{i, s}^{2}(n)\right\}+E\left\{\delta_{i, s}^{2}(m)\right\}+2 E\left\{\delta_{i, s}(n)\right\} E\left\{\delta_{i, s}(m)\right\},
\end{aligned}
$$

where we assume the quantization error is uncorrelated, and hence

$$
E\left\{\delta_{r, s}(n) \delta_{r, s}(m)\right\}=E\left\{\delta_{r, s}(n)\right\} E\left\{\delta_{r, s}(m)\right\} .
$$

Similarly, the mean squared error of the signal after complex subtraction becomes

$$
\begin{aligned}
& \sigma_{s+1, \text { sub }}^{2} \\
&= E\left\{\left|\left[\hat{x}_{r, s+1}(m)-x_{r, s+1}(m)\right]+j\left[\hat{x}_{i, s+1}(m)-x_{i, s+1}(m)\right]\right|^{2}\right\} \\
&= E\left\{\left|\left[\delta_{r, s}(n)-\delta_{r, s}(m)\right]+j\left[\delta_{i, s}(n)-\delta_{i, s}(m)\right]\right|^{2}\right\} \\
&= E\left\{\left|\delta_{r, s}(n)-\delta_{r, s}(m)\right|^{2}\right\}+E\left\{\left|\delta_{i, s}(n)-\delta_{i, s}(m)\right|^{2}\right\} \\
&= E\left\{\delta_{r, s}^{2}(n)\right\}+E\left\{\delta_{r, s}^{2}(m)\right\}-2 E\left\{\delta_{r, s}(n)\right\} E\left\{\delta_{r, s}(m)\right\} \\
&+E\left\{\delta_{i, s}^{2}(n)\right\}+E\left\{\delta_{i, s}^{2}(m)\right\}-2 E\left\{\delta_{i, s}(n)\right\} E\left\{\delta_{i, s}(m)\right\} .
\end{aligned}
$$

The mean squared error of all the signals at the stage $(s+1)$ can be calculated as

$$
\begin{aligned}
\sigma_{\mathrm{PE}, s+1}^{2} & =\frac{1}{2} \sigma_{s+1, \mathrm{add}}^{2}+\frac{1}{2} \sigma_{s+1, \mathrm{sub}}^{2} \\
& =2\left(E\left\{\delta_{r, s}^{2}(n)\right\}+E\left\{\delta_{i, s}^{2}(n)\right\}\right) \\
& =2 \sigma_{\mathrm{PE}, s}^{2} .
\end{aligned}
$$

\section{B. Derivation of Signal Energy at Each Stage}

For $r$-point DFT, Parseval's theorem states

$$
\sum_{n=0}^{r-1}|x(n)|^{2}=\frac{1}{r} \sum_{k=0}^{r-1}|X(k)|^{2} .
$$

Thus, for $N$-point FFT with $N=r^{\nu}$, the sum of the squared magnitude of the outputs at stage 1 can be rewritten as

$$
\begin{aligned}
\sum_{k=0}^{N-1}\left|x_{1}(k)\right|^{2} & =\sum_{k_{2}=0}^{N / r-1}\left(\sum_{k_{1}=0}^{r-1}\left|x_{1}\left(k_{1}+k_{2} r\right)\right|^{2}\right) \\
& =\sum_{n_{2}=0}^{N / r-1}\left(r \sum_{n_{1}=0}^{r-1}\left|x\left(n_{1} \frac{N}{r}+n_{2}\right)\right|^{2}\right) \\
& =r \sum_{n=0}^{N-1}|x(n)|^{2} .
\end{aligned}
$$

From the above, if an $\mathrm{N}$-point FFT is decomposed into $v$ stages of the $r$-point FFT, the sum of the squared magnitude of the outputs at the sth stage is given by

$$
\sum_{n=0}^{N-1}\left|x_{s}(n)\right|^{2}=r^{s} \sum_{n=0}^{N-1}|x(n)|^{2}=\frac{1}{r^{\nu-s}} \sum_{k=0}^{N-1}|X(k)|^{2} .
$$

In summary, for each stage of $r$-point FFT, the energy sum increases by $r$ times. Moreover, the magnitude of twiddle factors is all 1 , the existence of complex multiplications stages does not influence this result. 


\section{Acknowledgment}

This work was supported in part by the National Science Council, Taiwan, under Grants no. NSC 98-2220-E-008-004 and NSC 98-2220-E-008-001.

\section{References}

[1] Y. W. Lin, H. Y. Liu, and C. Y. Lee, "A dynamic scaling FFT processor for DVB-T applications," IEEE Journal of Solid-State Circuits, vol. 39, no. 11, pp. 2005-2013, 2004.

[2] K. Maharatna, E. Grass, and U. Jagdhold, "A 64-point Fourier transform chip for high-speed wireless LAN application using OFDM," IEEE Journal of Solid-State Circuits, vol. 39, no. 3, pp. 484-493, 2004.

[3] Y. W. Lin, H. Y. Liu, and C. Y. Lee, "A 1-GS/s FFT/IFFT processor for UWB applications," IEEE Journal of Solid-State Circuits, vol. 40, no. 8, pp. 1726-1735, 2005.

[4] Y.-T. Lin, P.-Y. Tsai, and T.-D. Chiueh, "Low-power variablelength fast Fourier transform processor," IEE Proceedings: Computers and Digital Techniques, vol. 152, no. 4, pp. 499-506, 2005.

[5] P. Y. Tsai, T. H. Lee, and T. D. Chiueh, "Power-efficient continuous-flow memory-base FFTprocessor for WiMAX OFDM mode," in Proceedings of the International Symposium on Intelligent Signal Processing and Communication Systems, December 2006.

[6] Y. W. Lin and C. Y. Lee, "Design of an FFT/IFFT processor for MIMO OFDM systems," IEEE Transactions on Circuits and Systems I, vol. 54, no. 4, pp. 807-815, 2007.

[7] Y. Chen, Y. W. Lin, Y. C. Tsao, and C. Y. Lee, "A 2.4-Gsample/s DVFS FFT processor for MIMO OFDM communication systems," IEEE Journal of Solid-State Circuits, vol. 43, no. 5, Article ID 4494644, pp. 1260-1273, 2008.

[8] T. J. Ding, J. V. McCanny, and YI. Hu, "Rapid design of application specific FFT cores," IEEE Transactions on Signal Processing, vol. 47, no. 5, pp. 1371-1381, 1999.

[9] A. Melnyk and B. Dunets, "FFT Processor IP Cores synthesis on the base of configurable pipelinearchitecture," in Proceedings of the International Conference on CAD Systems in Microelectronics (CADSM '03), pp. 211-213, February 2003.

[10] Xilinx, Inc., "Xilinx LogiCore: Fast Fourier Transform v6.0," product specification, Sepember 2008.

[11] H. Kee, N. Petersen, J. Kornerup, and S. S. Bhattacharyya, "Systematic generation of FPGA-based FFT implementations," in Proceedings of IEEE International Conference on Acoustics, Speech and Signal Processing (ICASSP '08), pp. 1413-1416, April 2008.

[12] G. Nordin, P. A. Milder, J. C. Hoe, and M. Püschel, "Automatic generation of customized discrete Fourier transform IPs," in Proceedings of the 42nd Design Automation Conference (DAC '05), pp. 471-474, June 2005.

[13] P. D'Alberto, P. A. Milder, A. Sandryhaila et al., "Generating FPGA-accelerated DFT libraries," in Proceedings of the 15th Annual IEEE Symposium on Field-Programmable Custom Computing Machines (FCCM '07), pp. 173-184, April 2007.

[14] T. H. Tsa and C. C. Peng, "A FFT/IFFT soft IP generator for OFDM communication system," in Proceedings of IEEE International Conference on Multimedia and Expo (ICME '04), pp. 241-244, June 2004.

[15] A. Cortés, I. Vélez, J. F. Sevillano, and A. Irizar, "An approach to simplify the design of IFFT/FFT cores for OFDM systems,"
IEEE Transactions on Consumer Electronics, vol. 52, no. 1, pp. 26-32, 2006.

[16] S. Saponara, N. E. L'Insalata, and L. Fanucci, "Low-complexity FFT/IFFT IP hardware macrocells for OFDM and MIMOOFDM CMOS transceivers," Microprocessors and Microsystems, vol. 33, no. 3, pp. 191-200, 2009.

[17] N. E. L'insalata, S. Saponara, L. Fanucci, and P. Terreni, "Automatic synthesis of cost effective FFT/FFT cores for VLSI OFDM systems," IEICE Transactions on Electronics, vol. E91-C, no. 4, pp. 487-496, 2008.

[18] N. Li and N. P. Van Der Meijs, "A radix $2^{2}$ based parallel pipeline FFT processor for MB-OFDM UWB system," in Proceedings of IEEE International SOC Conference (SOCC '09), pp. 383-385, September 2009.

[19] J. Lee, H. Lee, S. I. Cho, and S. S. Choi, "A high-speed, low-complexity radix- $2^{4}$ FFT processor for MB-OFDM UWB systems," in Proceedings of IEEE International Symposium on Circuits and Systems (ISCAS '06), pp. 4719-4722, May 2006.

[20] H. Lee and M. Shin, "A high-speed low-complexity twoparallel radix-24 FFT/IFFT processorfor MB-OFDM UWB systems," IEICE Transactions on Fundamentals of Electronics, Communications and Computer Sciences, vol. E91-A, pp. 12061211, 2008.

[21] S. Y. Lee, D. S. Kim, K. Y. Wang, B. S. Kim, and D. J. Chung, "Multi-input $\mathrm{r}^{3} \mathrm{SDF}-\mathrm{kR}$ for efficient FFT processor in MIMO-OFDM systems," IEICE Electronics Express, vol. 6, no. 24, pp. 1702-1707, 2009.

[22] BO. Fu and P. Ampadu, "An area efficient FFT/IFFT processor for MIMO-OFDM WLAN 802.11n," Journal of Signal Processing Systems, vol. 56, no. 1, pp. 59-68, 2009.

[23] M. Shin and H. Lee, "A high-speed four-parallel radix- $2^{4}$ FFT/IFFT processor for UWB applications," in Proceedings of IEEE International Symposium on Circuits and Systems (ISCAS '08), pp. 960-963, May 2008.

[24] R. S. Sherratt, O. Cadenas, and N. Goswami, "A low clock frequency FFT core implementation for multiband fullrate ultra-wideband (UWB) receivers," IEEE Transactions on Consumer Electronics, vol. 51, no. 3, pp. 798-802, 2005.

[25] C. Y. Wang, C. B. Kuo, and J. Y. Jou, "Hybrid word-length optimization methods of pipelined FFT processors," IEEE Transactions on Computers, vol. 56, no. 8, pp. 1105-1118, 2007.

[26] E. Bidet, D. Castelain, C. Joanblanq, and P. Senn, "A fast single-chip implementation of 8192 complex point FFT," IEEE Journal of Solid-State Circuits, vol. 30, no. 3, pp. 300-305, 1995.

[27] "Spiral software/hardware generation for DSP algorithms," http://www.spiral.net/hardware/dftgen.html.

[28] G. D. Wu and Y. M. Liu, "Radix-2 $2^{2}$ based low power reconfigurable FFT processor," in Proceedings of IEEE International Symposium on Industrial Electronics (ISIE '09), pp. 1134-1138, July 2009.

[29] S. Saponara and L. Fanucci, "VLSI design investigation for low-cost, low-power FFT/IFFT processing in advanced VDSL transceivers," Microelectronics Journal, vol. 34, no. 2, pp. 133$148,2003$. 